\title{
Modelling of Uncertainties using Fuzzy Interval for Enhancement of Images Corrupted by Impulse Noise
}

\author{
Rashmi Kumari \\ ECE Deptt \\ Galgotias University \\ Greater Noida, U.P., INDIA
}

\author{
S.K.Aggarwal \\ ECE Deptt. \\ JJT University \\ Jhunjhunu, Rajasthan, INDIA
}

\begin{abstract}
Noise filtering is the fundamental pre-processing step for digital images. In this paper we present a novel method in which the uncertainties of fuzzy membership function is modeled to reduce and the concept of this reduced uncertainties is used to detect the impulse corrupted pixels of digital images. Taking an interval instead of using a crisp value of membership function deals better with the uncertainties arises due to noisy data, uncertain meaning of word etc. Impulse noise is detected by using Laplacian operator and blurred S-shaped fuzzy membership function is used for removal of impulse noise where for the restoration the half of sum of mean and median of the kernel is used. The performance is compared with other existing filters on the basis of PSNR values calculated for original and restored images.
\end{abstract}

\section{General Terms}

Fuzzy Logic, S-Shaped membership function, Fuzzy filtering, Grayscale image

\section{Keywords}

Type-2 Fuzzy Logic System, Impulse Noise Removal, Image Processing

\section{INTRODUCTION}

Noise reduction is still the challenging problem in digital image processing. In digital image impulse noise identification and reduction is an important pre-processing step as images are often corrupted by noise during acquisition and transmission process. In the literature several fuzzy and non-fuzzy filters[1] have been studied for impulse noise reduction, in which one of the more famous filter for gray scale image is the median filter[2] where the central pixel intensity is replaced by a median value taken within a filtering window. The median filter is very simple and computationally efficient but it causes the loss of fine image details because it replaces all the pixels of the noisy image by a median value irrespective of them being corrupted or not.

To overcome the disadvantages of median filtering the solution is to detect first the corrupted pixel and then to apply filtering operation on those pixels. One of the main problems with impulse noise detection is that it is difficult to differentiate between an edge and an impulse noise because the both are having peaks in their neighbourhood in the intensity space.

In the literature several modified medians filters are studied for impulse noise removal including the switching median filter(SM) [6], the centre weighted median filter [3], the RCM filter [4], the tri-state median filter(TSM) [5], the multi-state median filter(MSM), adaptive two pass rank order filter,
Improved median filter etc. Several fuzzy filters [7] - [13] such as fuzzy median filter (FMF), fuzzy weighted mean filter, adaptive weighted mean filter (AWMF), FIRE filters, fuzzy thresholding filter(FTF), fuzzy switching filter(FSF), etc. have also been studied for the removal of impulse noise.

This paper presents a two step method to remove impulse noise from digital images. First the detection process will be based on the grayscale neighbourhood information and then fuzzy interval is used to remove the noise using S-shaped membership function where we find the extent of impulsiveness in more effective way. The paper is organized in this way that section 2 describes the type- 2 fuzzy sets and section 3 says about the filtering process. Section 4 contains the experimental result and section 5 is the conclusions of the above said process.

\section{UNCERTAINTIES MODELING BY FUZZY INTERVAL}

The type-2 fuzzy set is introduced by Zadeh as an extension of type-1 fuzzy set [14-16] which is based upon the concept of taking an interval instead of a crisp value of membership function. Figure 1(a) shows the interval taken for crisp value 1.3 as $1.25-1.35$ and figure 1(b) and 1(c) clearly depicts how the fuzzy number 1.3 represented by triangular membership function is modeled by taking an interval between the number 1.28 and 1.32. It minimizes the effect of uncertainties in rule based fuzzy logic systems (FLS). It can be useful when the decision of accurate membership function is difficult due to uncertainties in shape, location or other parameters.

For type-1 fuzzy logic systems, there are basically four sources of uncertainties have been identified.
(a) Uncertain meaning of words
(b) Consequences associated with a histogram of values
(c) Uncertain measurements
(d) Noisy data

The impact of these factors is as an uncertain membership function and type-1 fuzzy sets have crisp membership function that is insufficient to model these uncertainties. 

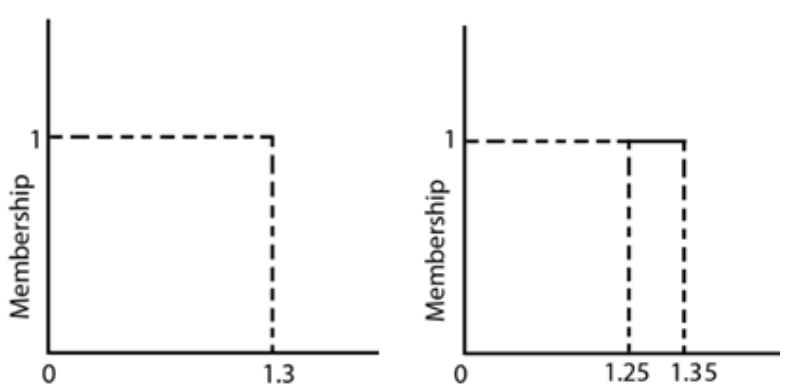

Figure 1(a) Crisp value interval around point 1.3

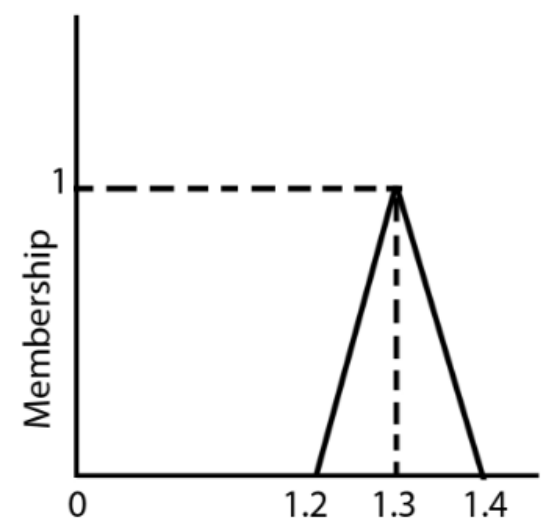

Figure 1(b) Fuzzy membership function

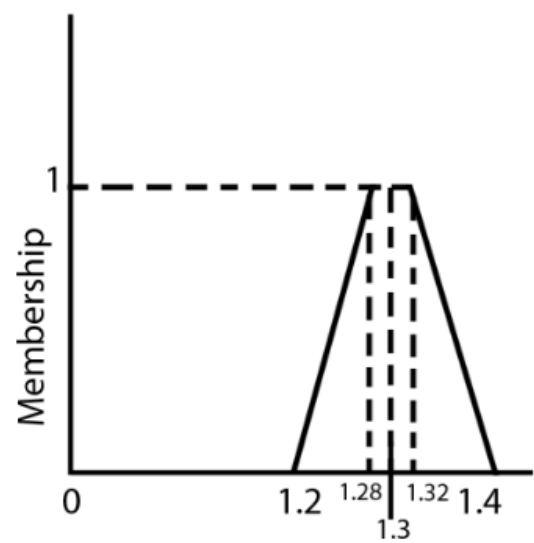

Figure 1(c) Fuzzy mfs interval around point 1.3

Type-1 fuzzy sets is modelled by two dimensional membership function where as the type- 2 is modelled by three dimensional membership function (figure 2). This third dimension allows the additional degree of freedom by which uncertainties can be modelled in a better way.

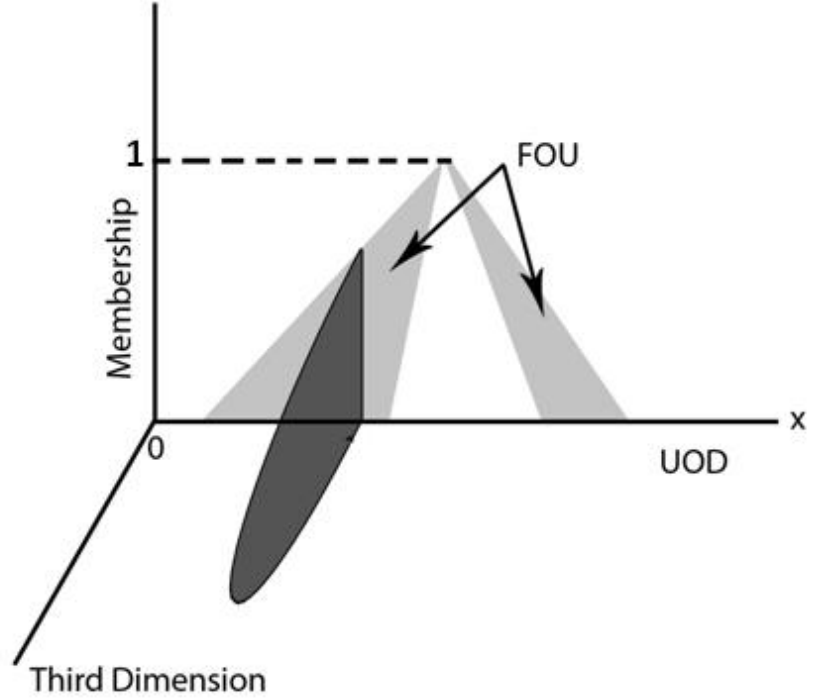

Figure 2. Third dimension of type -2 fuzzy set.

Membership function of type-2 fuzzy set can be obtained by blurring the membership function of type-1 fuzzy set [15]. Figure 3 shows the membership function of type- 1 fuzzy set where for $r=r^{\prime}$, the membership function $\mu\left(r^{\prime}\right)=u^{\prime}$. This value of the membership function is a crisp value. Whereas in figure 4 for $r=r$ ', the membership function takes all the values where the vertical line intersects the blur. This allows us to characterize a type- 2 fuzzy set to create a three dimensional membership functions.

Type-2 fuzzy set A is characterized as :

$$
\widetilde{A}=\left\{\left((x, u), \mu_{\tilde{A}}(x, u)\right) \mid \forall u \in J_{x} \subseteq[0,1]\right\}
$$

In which $0 \leq \mu_{\tilde{A}}(x, u) \leq 1$

Here $J x$ is the primary membership of $x$. The footprint of uncertainty (FOU) is expressed as

$$
\mathrm{FOU}=\bigcup_{x \in X} j_{x}
$$

It represents the uncertainty in the primary memberships of type-2 fuzzy set A and it is the union of all primary membership functions as shown by shaded region of figure 4 . So, the membership functions of type-2 fuzzy sets give us an interval instead of a single value for every element.

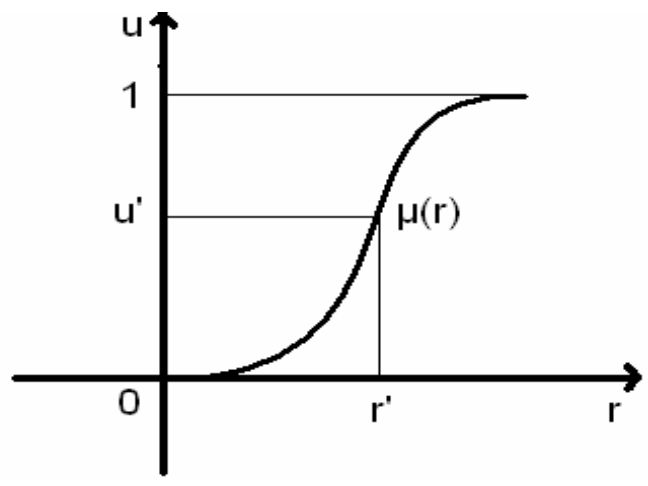

Figure 3. Membership function of a type-I fuzzy set. 


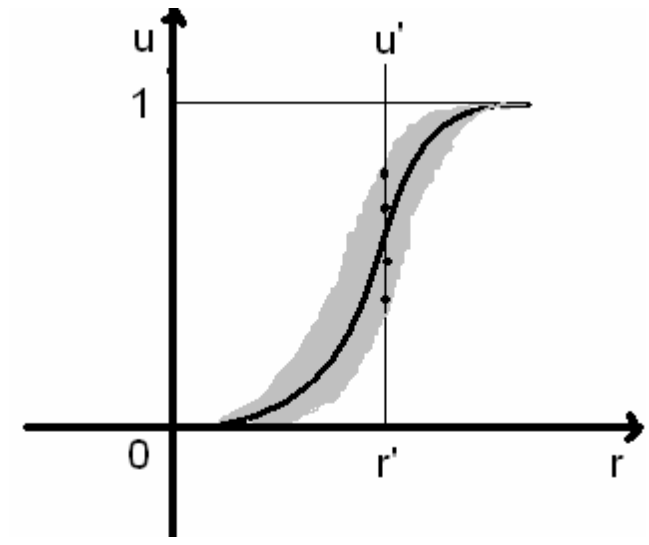

Figure 4. Membership function of a type-II fuzzy set.

\section{FILTERING TECHNIQUES}

This section presents the filtering technique based on type-2 fuzzy interval concepts. This technique is a two-step process in which first we will detect the pixel is noisy or not and then filtering operation can be performed on that noisy pixels where impulsive pixels modifies on basis of type-2 fuzzy switching median scheme.

\subsection{Impulse Noise Detection}

The impulse detection is usually based on the following two assumptions [16]: (a) a noise-free image consists of locally smoothly varying areas separated by edges; (b) a noise pixel takes a grayscale value substantially larger or smaller than those of its neighbours. First the filter window of size $5 \times 5$ is taken where center pixel is $I(i, j)$, and it is convolved with four one dimension Laplacian operators as shown in figure 5 respectively. Each of these operators gives the value of edges in a different orientation.

\begin{tabular}{|l|l|l|l|l|}
\hline 0 & 0 & 0 & 0 & 0 \\
\hline 0 & 0 & 0 & 0 & 0 \\
\hline-1 & -1 & 4 & -1 & -1 \\
\hline 0 & 0 & 0 & 0 & 0 \\
\hline 0 & 0 & 0 & 0 & 0 \\
\hline
\end{tabular}

\begin{tabular}{|l|l|l|l|l|}
\hline-1 & 0 & 0 & 0 & 0 \\
\hline 0 & -1 & 0 & 0 & 0 \\
\hline 0 & 0 & 4 & 0 & 0 \\
\hline 0 & 0 & 0 & -1 & 0 \\
\hline 0 & 0 & 0 & 0 & -1 \\
\hline
\end{tabular}

\begin{tabular}{|l|l|l|l|l|}
\hline 0 & 0 & 0 & 0 & -1 \\
\hline 0 & 0 & 0 & -1 & 0 \\
\hline 0 & 0 & 4 & 0 & 0 \\
\hline 0 & -1 & 0 & 0 & 0 \\
\hline-1 & 0 & 0 & 0 & 0 \\
\hline
\end{tabular}

\begin{tabular}{|l|l|l|l|l|}
\hline 0 & 0 & -1 & 0 & 0 \\
\hline 0 & 0 & -1 & 0 & 0 \\
\hline 0 & 0 & 4 & 0 & 0 \\
\hline 0 & 0 & -1 & 0 & 0 \\
\hline 0 & 0 & -1 & 0 & 0 \\
\hline
\end{tabular}

Figure 5. Four $5 \times 5$ convolution mask
For impulse noise detection we will take the minimum of all these four convolution as:

$$
r(i, j)=\min \left\{\left|I(i, j) * K_{p}\right|: 1-4\right\}
$$

Where $K_{p}$ is the pth kernel. The impulse can be detected by the analysing of the values of $r(i, j)$. The value of $r(i, j)$ is large when $I(i, j)$ is corrupted by noise, and $r(i, j)$ is small when $I(i, j)$ is noise-free whether or not it is a flat region, edge, or thin line pixels.

\subsection{Impulse Noise Detection Using Type-2 Fuzzy Logic}

Let $\mu[r(i, j)] \in[0,1]$ be the type-1 membership function of $r(i, j)$ which indicates the extent of the impulsiveness of the pixel $I(i, j)$. The following fuzzy rules [15] are applied:

Rule 1: If $r(i, j)$ is large, then $\mu[r(i, j)]$ is large.

Rule 2: If $r(i, j)$ is small, then $\mu[r(i, j)]$ is small

The S-shaped membership function is used to describe the type-1 membership function of the impulse noise corruption extent on the basis of these rules.

$\mu[r(i, j)]= \begin{cases}0 & \text { if } r(i, j) \leq \alpha \\ 2\left(\frac{r(i, j)-\alpha}{\gamma-\alpha}\right)^{2} & \text { if } \alpha \leq r(i, j) \leq \beta \\ 1-2\left(\frac{r(i, j)-\gamma}{\gamma-\alpha}\right)^{2} & \text { if } \beta \leq r(i, j) \leq \gamma \\ 1 & \text { if } r(i, j) \geq \gamma\end{cases}$

Where $\beta=(\alpha+\lambda) / 2$. 


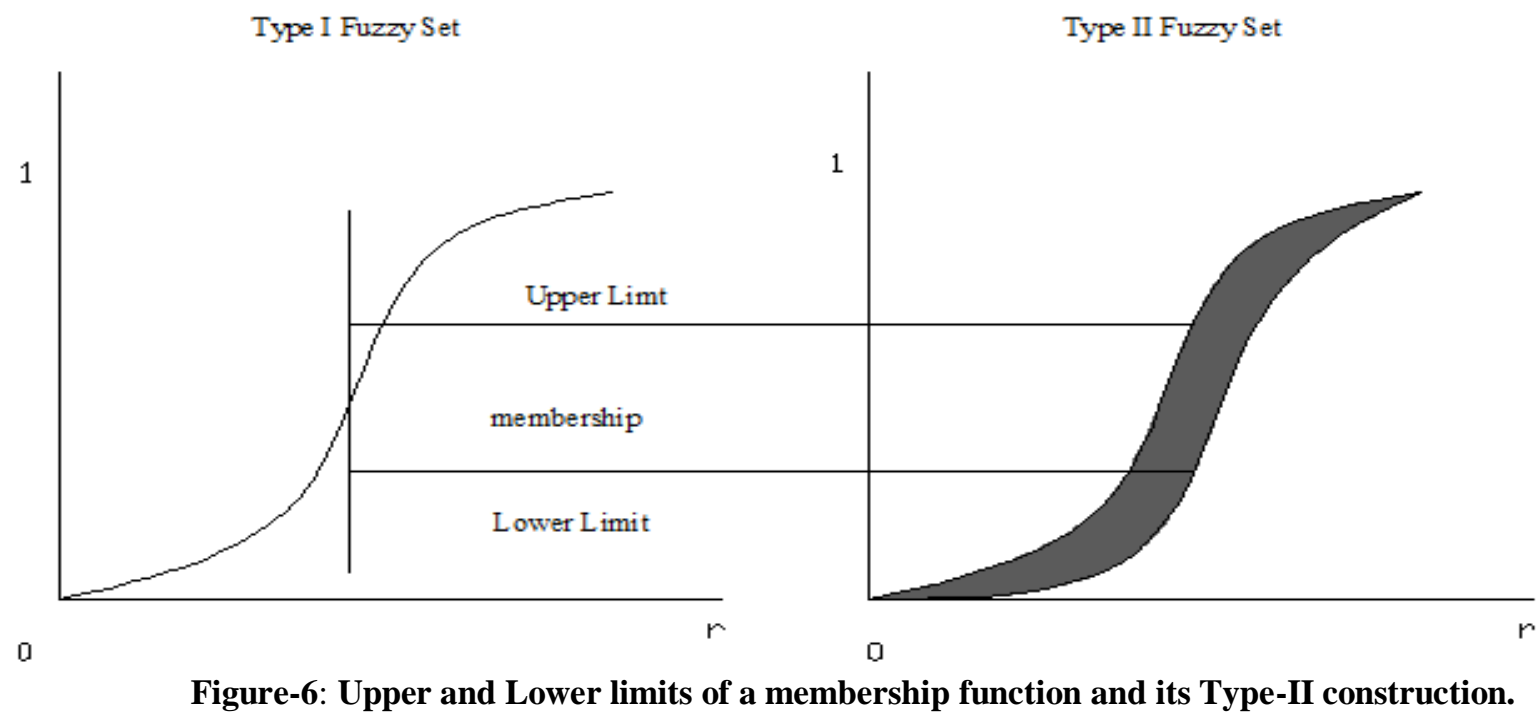

Type-2 fuzzy set is obtained by blurring the type-1 membership function. We simply assign the upper and lower limit to each element to construct the FOU as shown in figure 6. So Type-2 fuzzy set A is defined as:

$$
\begin{aligned}
\tilde{A}= & \left\{\left(x, \mu_{U}(x), \mu_{L}(x)\right) \mid \forall x \in X,\right. \\
& \left.\mu_{L}(x) \leq \mu(x) \leq \mu_{U}(x), \mu \in[0,1]\right\}
\end{aligned}
$$

The upper and lower membership degrees of the initial membership function $\mu$ can be defined as [20]-

$$
\begin{aligned}
& \mu_{\text {upper }}(x)=(\mu(x))^{0.5} \\
& \mu_{\text {lower }}(x)=(\mu(x))^{2}
\end{aligned}
$$

Where $0 \leq \mu(x) \leq 1$.

Here the uncertainties in the shape and position of the type-1 fuzzy set can be represented by means of the third dimension represented by the FOU.

The type-2 membership function is:

$$
\mu_{T 2}[r(i, j)]=\left(\mu_{\text {upper }} \times \delta\right)+\left(\mu_{\text {lower }} \times(1-\delta)\right)
$$

Where $0 \leq \delta \leq 1$

$$
\delta=\left(\left|g_{\text {mean }}-(L / 2)\right|\right) /(L / 2)
$$

Where $g_{\text {mean }}$ is the mean value of each sub-image and $\mathrm{L}$ is the number of gray levels used. The idea behind the $\mu_{\mathrm{T} 2}$ equation is that pixels with pixel values in a darker or a lighter neighbourhood will have greater proportion of $\mu_{\text {upper }}$ than

$$
\mu_{\text {lower }} \text {. }
$$

\subsection{Impulse Noise Removal}

The detected pixels are restored by using switching method in which only good pixels are considered to calculate the median value. Here a flag is set for the corrupted pixels for both kind of impulse noise as shown in figure 7 and a flag image sequence is generated (figure 8)[19].

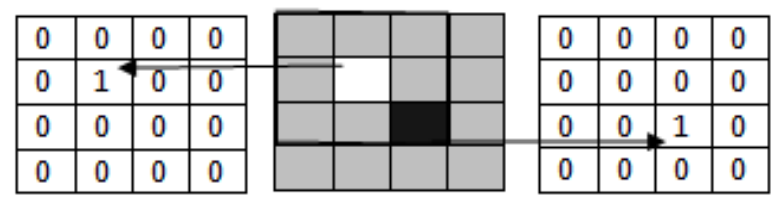

Figure 7. $3 \times 3$ window showing impulse in flag sequence

\begin{tabular}{|l|l|l|l|}
\hline 0 & 0 & 0 & 0 \\
\hline 0 & 1 & 0 & 0 \\
\hline 0 & 0 & 1 & 0 \\
\hline 0 & 0 & 0 & 0 \\
\hline
\end{tabular}

Figure 8. Final flag sequence

Now the corrupted pixels are restored by the value calculated as half of sum of mean value of uncorrupted pixels and median value of the uncorrupted pixels which can be viewed by the flag sequence image of corrupted pixels.

Suppose $y_{(i, j)}$ represents the pixel value at position $(\mathrm{i}, \mathrm{j}$ ) in the image and $f_{(i, j)}$ is used to indicate whether the pixel at position $(i, j)$ is impulse or not i.e. the flag sequence. Only good uncorrupted pixels are choosen to calculate the replaced value. Our method is substituting the corrupted pixels to the half of the sum of its median value $m_{(i, j)}$ and mean value $\bar{m}_{(i, j)}$ as defined as :

$$
S_{(i, j)}=\frac{1}{2}\left(m_{(i, j)}+\bar{m}_{(i, j)}\right)
$$


Finally the restored output is calculated by using the typeII membership value and the replaced pixel value $S(i, j)$ by using following relation.

$$
Y(i, j)=\left(\mu_{T 2} \times S_{(i, j)}\right)+\left(I(i, j) \times\left(1-\mu_{T 2}\right)\right)
$$

The output computed by this equation has improved result as compared with other fuzzy filters.

\section{EXPERIMENTAL RESULTS}

The performance of the filters are calculated and measured in terms of PSNR which is defined as follows:

\subsection{Root Mean Squared Error (RMSE)}

Suppose that the original image u of size M X N has been denoised, using an image denoising scheme, and let $\mathrm{u} \sim$ be the denoised estimate. The RMSE between the denoised image and the original image is given by:

$R M S E=\sqrt{\frac{\sum_{i=1}^{M} \sum_{j=1}^{N}\left(u i_{j}-u_{\sim i j}\right) 2}{M x N}}$

\subsection{Peak Signal to Noise Ratio (PSNR)}

It is inversely proportional to the RMSE, its units are in decibels $(\mathrm{dB})$ and is formally defined by

$$
P S N R=20 \log \left[\frac{255}{R M S E}\right](\mathrm{dB})
$$

Where value 255 is the maximum value of pixel for an 8 bits/pixel gray-scale image.

Three standard images( figure 9) are used to compute the PSNR for restored images and its comparative results are shown in Table 1.

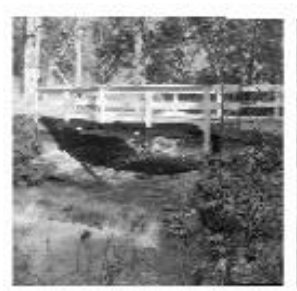

(a)

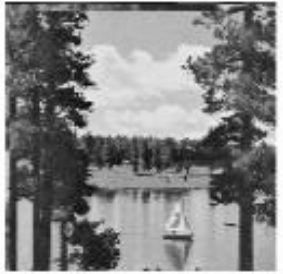

(b)

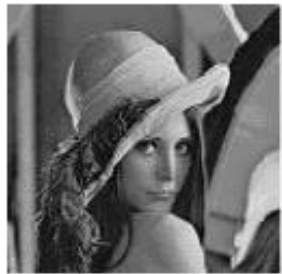

(c)
Figure 9. Standard images (a) Bridge (b) Lake (c) Lena

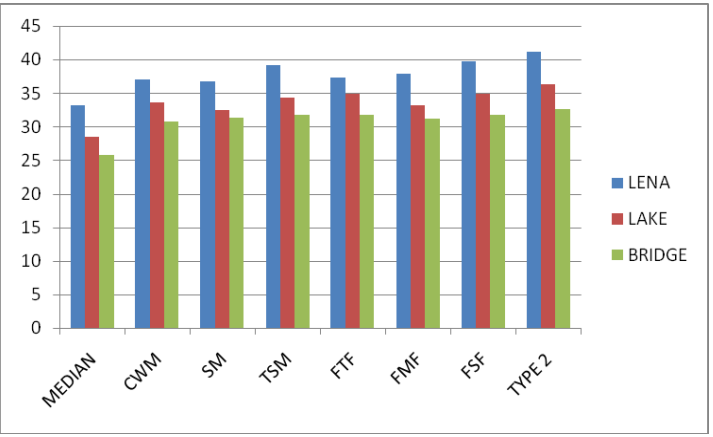

Figure 10. Computed PSNR of filters for 5\% impulse noise

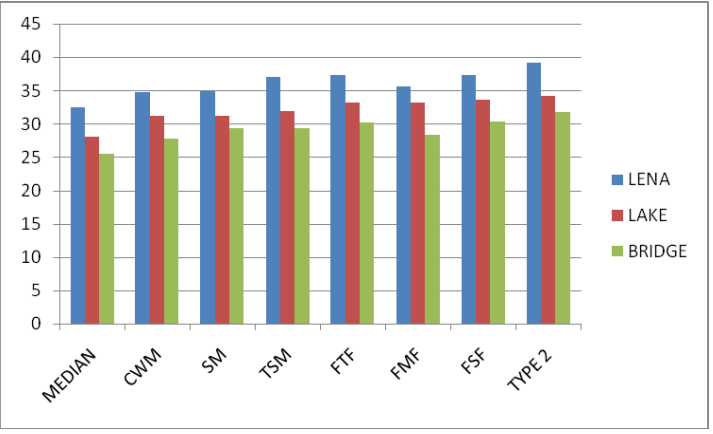

Figure 11. Computed PSNR of filters for $10 \%$ impulse noise

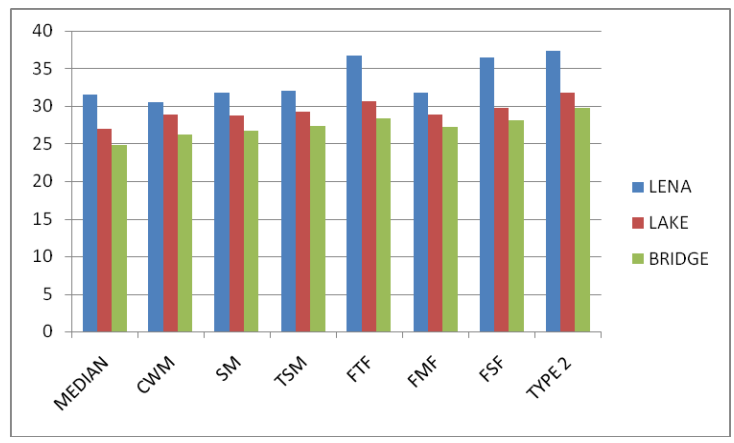

Figure 12. Computed PSNR of filters for $20 \%$ impulse noise

\section{CONCLUSIONS}

A fuzzy filter has been designed by using fuzzy interval or by blurring the primary membership function. The corrupted image is restored by taking the fuzzy interval grade value and the half of the sum of mean and median of uncorrupted pixels of window mask for impulse noise detection and removal from digital images. The simulation results shows that the result of this proposed filter is better than other existing filters. This algorithm can be modified and applied for the higher noise ratios as well as for colour images. 
Table 1: Comparative results in PSNR of different filtering methods for three standard images for 5\%, $10 \%$ and $20 \%$ impulse noise ratio

\begin{tabular}{|c|c|c|c|c|c|c|c|c|c|}
\hline \multirow{3}{*}{ FILTERS } & & \multirow{2}{*}{\multicolumn{3}{|c|}{ 20\% IMPULSE NOISE }} \\
\hline & \multicolumn{3}{|c|}{$5 \%$ IMPULSE NOISE } & \multicolumn{3}{|c|}{$10 \%$ IMPULSE NOISE } & & & \\
\hline & LENA & LAKE & BRIDGE & LENA & LAKE & BRIDGE & LENA & LAKE & BRIDGE \\
\hline MEDIAN & 33.2 & 28.54 & 25.78 & 32.5 & 28.12 & 25.5 & 31.5 & 26.9 & 24.8 \\
\hline CWM & 37.0 & 33.61 & 30.69 & 34.8 & 31.2 & 27.82 & 30.5 & 28.8 & 26.2 \\
\hline SM & 36.7 & 32.44 & 31.28 & 34.9 & 31.12 & 29.28 & 31.8 & 28.76 & 26.68 \\
\hline TSM & 39.1 & 34.26 & 31.8 & 37.0 & 31.83 & 29.32 & 32.0 & 29.23 & 27.28 \\
\hline FTF & 37.29 & 34.86 & 31.75 & 37.36 & 33.23 & 30.2 & 36.76 & 30.6 & 28.32 \\
\hline FMF & 37.9 & 33.25 & 31.23 & 35.6 & 33.24 & 28.4 & 31.8 & 28.9 & 27.2 \\
\hline FSF & 39.8 & 34.92 & 31.8 & 37.36 & 33.6 & 30.34 & 36.4 & 29.8 & 28.1 \\
\hline TYPE 2 & 41.2 & 36.28 & 32.62 & 39.2 & 34.23 & 31.69 & 37.36 & 31.8 & 29.75 \\
\hline
\end{tabular}

\section{REFERENCES}

[1] R. Gonzalez and R.Woods, 2008, Digital Image Processing, PHI II Edition.

[2] S-J. Ko and Y. H. Lee, Sept 1991,“Centre-weighted median filters and their applications to image enhancement" IEEE Trans. Circuits and Syst., vol. 38, pp. 984-993.

[3] L. Alparone, S. Baronti and R. Carla, Feb. 1995, "Two Dimensional Rank Conditioned Median Filter ," IEEE Trans. On Circuits and systems - II : vol 42, No.2.

[4] T. Sun and Y. Neuvo , Apr. 1994, "Detail preserving median based filters in image processing," Pattern Recognit. Lett., vol. 15 , pp 341-347.

[5] T. Chen, K. K. Ma, L.H. Chen, Dec. 1999, "Tri-state median filter for image denoising," IEEE Trans. Image Processing, vol. 8, pp. 1834-1838.

[6] Zhang, S. Karim, M. A., 2002, “A New Impulse Detector for Switching Median Filter", IEEE Signal Processing Lett., vol. 9, pp. 360-363.

[7] F. Russo and G. Ramponi, June 1996, “A fuzzy filter for images corrupted by impulse noise," IEEE Signal Process. Lett. Vol. 3, no. 6, pp. 168-170.

[8] F. Russo , Apr. 1999, "Fire operators for image processing," Fuzzy Sets Syst., vol. 103, pp. 265-275.

[9] C. S. Lee, Y. H. Kuo, and P.T. Yu , Jul. 1997, "Weighted fuzzy mean filters for image processing," Fuzzy Sets Syst., vol. 89, pp. 157-180.

[10] C. S. Lee, Y. H. Kuo,2000, “Adaptive fuzzy filter and its application to image enhancement," in Fuzzy techniques in Image Processing, I edition E. E. Kerre and M. Nachtegael, Eds. , Heidelberg, germany: Physica Verlag, vol. 52, pp. 172-193.
[11] S. Schulte, M. Nachtegael, V.D.Witte D.V.Weken, E.E. Kerre.,May 2006, “ A Fuzzy impulse noise detection and reduction method.," IEEE Trans. On Image Processing.

[12] H.Xu, G. Zhu, H. Peng, D. Wang , April 2004, "Adaptive fuzzy switching filter for images corrupted by impulse noise ," Pattern Recognit. Lett., vol. 25 , pp 1657-1663.

[13] J. C. Sheng Y. J. Runtao, 2000, "Fuzzy weighted average filter" In Proc. ICSP 2000, pp. 525-528.

[14] Mendel, J. M., John, R.I.B., April 2002, “Type-2 Fuzzy Sets Made Simple". IEEE Transactions on Fuzzy Systems, vol. 10, no. 2, pp.117-27.

[15] Karnik, N.N., Mendel, J. M., 1998, "Introduction to Type-2 Fuzzy Logic Systems",IEEE World Congress on Computational Intelligence, vol.2, p 915- 935.

[16] H. R. Tizhoosh, 2005, "Image Thresholding using TypeII Fuzzy Sets", Pattern Recognition vol. 38, pp 23632372.

[17] M. T. Yildirim, A. Basturk, 2007, “A Detail Preserving Type-2 Fuzzy Logic Filter for Impulse Noise removal from Digital Images", Fuzzy Systems Conference, FUZZ-IEEE.

[18] J. M. Mendel, R. I. John, F. Liu, Dec. 2006, "Interval Type-II Fuzzy Logic Systems Made Simple”, IEEE Transactions on Fuzzy Systems, vol. 14.

[19] Soon Ting Boo, H. Ibrahim, Kanny Kol Vin Toh, 2009, "An Improved Progressive Switching Median Filter", IEEE Intenational conference on future computer and communication, Malaysia.

[20] Rashmi Kumari, S.K.Aggarwal, March 2012, "Impulse Noise Removal Using Type-II Fuzzy Sets",Procc. of Information Systems \& Computer Networks, pp.23-26. 